\title{
Real-Time Assessment of Staphylococcus aureus Biofilm Disruption by Phage-Derived Proteins
}

\author{
Diana Gutiérrez*, Lucía Fernández, Beatriz Martínez, Patricia Ruas-Madiedo, \\ Pilar García and Ana Rodríguez
}

Instituto de Productos Lácteos de Asturias, Consejo Superior de Investigaciones Científicas, Villaviciosa, Spain

\section{OPEN ACCESS}

Edited by:

Heather K. Allen,

National Animal Disease Center,

United States

Reviewed by:

César de la Fuente,

Massachusetts Institute

of Technology, United States

Mathias Schmelcher,

ETH Zurich, Switzerland

${ }^{*}$ Correspondence:

Diana Gutiérrez

dianagufer@ipla.csic.es

Specialty section:

This article was submitted to Antimicrobials, Resistance

and Chemotherapy,

a section of the journa

Frontiers in Microbiology

Received: 07 June 2017 Accepted: 11 August 2017 Published: 24 August 2017

Citation

Gutiérrez $D$, Fernández $L$, Martínez B, Ruas-Madiedo $P$, García P and Rodríguez A (2017)

Real-Time Assessment of Staphylococcus aureus Biofilm

Disruption by Phage-Derived

Proteins. Front. Microbiol. 8:1632. doi: 10.3389/fmicb.2017.01632
A current focus of research is the development of new tools for removing bacterial biofilms in industrial settings. Bacteriophage-encoded proteins, such as endolysins, virion-associated peptidoglycan hydrolases, and exopolysaccharide depolymerases, have been shown to be efficient against these structures. However, the current screening techniques for the identification of antibiofilm properties of phage-derived proteins have important shortcomings. The aim of this work was to use the rapid, reproducible and accurate technology "real-time cell analyzer" for screening and comparing the antibiofilm ability of four phage-derived compounds, three lytic proteins (LysH5, CHAP-SH3b, and HydH5-SH3b) and one exopolysaccharide depolymerase (Dpo7) against Staphylococcus aureus biofilms, which have been associated with recurrent contamination of food products. The data generated after biofilm treatment allowed for the calculation of different antibiofilm parameters: (1) the minimum biofilm eradicating concentration that removes $50 \%$ of the biofilm (ranging from $3.5 \pm 1.1$ to $6.6 \pm 0.5 \mu \mathrm{M})$, (2) the lowest concentration needed to observe an antibiofilm effect ( $\sim 1.5 \mu \mathrm{M}$ for all the proteins), and (3) the specific antibiofilm activity and the percentage of biofilm removal that revealed LysH5 as the best antibiofilm compound. Overall, this technology might be used to quickly assess and compare by standardized parameters the disaggregating activity of phage antibiofilm proteins. Keywords: biofilm, Staphylococcus aureus, RTCA, MBEC 50 , LOABE, specific antibiofilm activity, phage lytic
proteins, exopolysaccharide depolymerase

\section{INTRODUCTION}

In most environments, bacterial cells are commonly organized into mono or multi-species biofilms attached to a surface. These sessile communities generally have a specific tridimensional structure where the cells are entrapped in a matrix that can be composed of polysaccharides, proteins, teichoic acids, nucleic acids, and lipids (Flemming and Wingender, 2010). This particular lifestyle confers several advantages on the bacterial cells that ultimately enhance their tolerance to harsh environmental conditions. Thus, bacteria adhered to an inert surface or a tissue can avoid being washed away by water flow or the bloodstream and, perhaps more importantly, they are more resistant or tolerant to antibiotics, biocides and host defense mechanisms (Bridier et al., 2015; Olsen, 2015). 
Bacterial biofilms have a huge impact on the environment, human health, and a wide variety of industrial processes (Hall-Stoodley and Stoodley, 2009; Van Houdt and Michiels, 2010; Martin et al., 2016). In fact, according to the National Institutes of Health, biofilms are responsible for more than $60 \%$ of microbial infections in humans and $80 \%$ of chronic infections (Bjarnsholt, 2013). Furthermore, these structures can cause severe economic losses in the food industry due to corrosion or obstruction of equipment, reduction in heat transfer and recurrent contamination of food products by spoilage or pathogenic bacteria, which represent an additional risk for public health (Myszka and Czaczyk, 2011). The resistance of biofilms to sanitation processes (Anand and Singh, 2013) together with a poor hygiene of food contact surfaces and equipment has repeatedly led to recurrent contamination of food products that can cause food-borne disease outbreaks. Particularly, the foodborne outbreaks related to the Staphylococcus aureus enterotoxins represented $6.4 \%$ of all the outbreaks in the EU in 2014 (EFSA and ECDC, 2016). Indeed, the ability to form biofilms enables this bacterium to colonize not only abiotic surfaces but also human tissues, making $S$. aureus one of the most important causative agents of nosocomial infections related to implanted medical devices (ECDC, 2013; Otto, 2013). The extracellular matrix of staphylococcal biofilms usually contains the exopolysaccharide poly- $\beta$-(1-6)- $N$-acetyl-glucosamine (PIA/PNAG), but biofilms lacking this component can be formed by the mediation of different surface proteins such as SasC, SasG, the clumping factor $\mathrm{B}(\mathrm{ClfB})$, the serine aspartate repeat protein $(\mathrm{SdrC})$, the biofilm-associated protein (Bap), and the fibronectin/fibrinogenbinding proteins (FnBPA and FnBPB) (Speziale et al., 2014). Extracellular DNA (eDNA) is also an important component of the staphylococcal biofilm matrix and can participate in horizontal gene transfer (Montanaro et al., 2011).

Taking into account the serious human health risk posed by biofilms formed by pathogenic bacteria, it is imperative to design new antibiofilm strategies. A major goal is to develop novel antimicrobials that can circumvent biofilm resistance mechanisms, which include limited penetration of antimicrobial agents, reduced cell growth rate inside the biofilm and the presence of antimicrobial-tolerant persister cells, to name a few (Van Acker et al., 2014). Ideally, an antibiofilm agent should be able to penetrate the biofilm structure, degrade the matrix, and eliminate the bacteria (Rabin et al., 2015). This can be achieved by following two different approaches, which are inhibition of bacterial surface attachment and destabilization/disruption of mature biofilms (Miquel et al., 2016). Within this context, the use of bacteriophages represents a promising strategy to prevent and/or eliminate bacterial biofilms (Gutiérrez et al., 2016b). Bacteriophages are viruses that specifically target bacteria while being harmless to humans, animals and plants. The relentless rise in the antibiotic resistance of pathogenic bacteria has boosted a renewed interest in the utilization of phages as antimicrobials. Indeed, phages have been successfully used to fight against pathogenic bacteria in clinical, veterinary, food safety, and environmental contexts (O'Flaherty et al., 2009; García et al., 2010) and have shown promising results as antibiofilm agents (Gutiérrez et al., 2016b). Moreover, bacteriophages also constitute a source of novel antimicrobial enzymes that can be exploited to combat bacterial biofilms. For instance, phage-encoded lytic proteins, such as endolysins and virion-associated peptidoglycan hydrolases (VAPGHs), have shown great promise as antimicrobial agents (Rodríguez-Rubio et al., 2015). Furthermore, some authors have demonstrated that endolysins display antibiofilm activity (Sass and Bierbaum, 2007; Shen et al., 2013; Oliveira et al., 2014) and, in some cases, have proven their ability to kill persister cells (Gutiérrez et al., 2014). Additionally, phage-encoded proteins with polysaccharide depolymerase activity can be used for biofilm removal due to their ability to degrade the polysaccharide matrix (Gutiérrez et al., 2015; Pires et al., 2016).

Nowadays, the development of new antibiofilm agents can be hindered by the need for a reliable and accurate technology that allows the screening of the activity of these proteins. In a previous work, we demonstrated and validated that the xCelligence real-time cell analyzer (RTCA) equipment can be used to monitor biofilm formation and disruption in different bacterial species (Gutiérrez et al., 2016a). This was subsequently verified by Ferrer et al. (2017). This method offers several advantages compared to other techniques commonly used to test potential antibiofilm agents, like crystal violet staining or viable cell counting. Indeed, this system, which is based on impedance measurement recorded as cell index (CI), is non-invasive, labelfree, fast, and reproducible. Here, we have used this technology to monitor biofilm elimination by different phage-derived proteins, in order to provide a rapid and standardized method to define and compare antibiofilm activities using different parameters such as minimum biofilm eradicating concentration that removes $50 \%$ of the biofilm $\left(\mathrm{MBEC}_{50}\right)$, lowest observed antibiofilm effect (LOABE), specific antibiofilm activity, and percentage of biofilm removal.

\section{MATERIALS AND METHODS}

\section{Bacterial Strains, Culture Conditions, and Proteins}

Four S. aureus strains (15981, ISP479r, IPLA1, and Sa9) were selected to test the antibiofilm activity of phage-derived proteins. S. aureus 15981 and ISP479r have a clinical origin (Valle et al., 2003), while $S$. aureus IPLA1 and Sa9 were isolated from food environments (García et al., 2007; Gutiérrez et al., 2012). As standard culture conditions, strains were grown in TSB (Tryptic Soy Broth, Scharlau, Barcelona, Spain) at $37^{\circ} \mathrm{C}$ with shaking, and frozen stocks (stored at $-80^{\circ} \mathrm{C}$ ) were plated onto TSB supplemented with $2 \%$ agar (TSA) and incubated for $24 \mathrm{~h}$.

Phage-derived proteins LysH5 (García et al., 2010), CHAPSH3b, HydH5-SH3b (Rodríguez-Rubio et al., 2012b), and Dpo7 (Gutiérrez et al., 2015) were purified as previously described. Prior to biofilm treatment assays, the buffer was exchanged to $50 \mathrm{mM}$ sodium phosphate $(\mathrm{NaPi})$ buffer $(\mathrm{pH}=7.4)$ using the Kit "Zeba TM Spin Desalting Columns, 7K MWCO, $5 \mathrm{~mL}$ " (Thermo Fisher Scientific, Madrid, Spain) following the supplier's recommendations; afterward, the supernatants were filtered using $0.22 \mu \mathrm{m}$ PES membrane filters (VWR, Spain). The amount 
of protein was quantified by using the Quick Start ${ }^{\mathrm{TM}}$ Bradford Protein Assay kit (Bio-Rad, Madrid, Spain). Quantification of the lytic activity of proteins LysH5, CHAP-SH3b, and HydH5$\mathrm{SH} 3 \mathrm{~b}$ was performed by the turbidity reduction assay against planktonic S. aureus Sa9 cells (Obeso et al., 2008) and by MIC (minimal inhibitory concentration) determination assay which was carried out by the conventional broth microdilution technique in TSB (CLSI, 2015). The specific lytic activity of the lytic proteins was calculated as the decrease in the absorbance $\left(\mathrm{OD}_{600} \mathrm{~nm}\right)$ per $\mu \mathrm{M}$ of protein and per minute $\left(\Delta \mathrm{OD} \times \mu \mathrm{M}^{-1} \times \mathrm{min}^{-1}\right)$; the MIC was set at the concentration $(\mu \mathrm{M})$ where no bacterial growth was observed and calculated as the mode of three independent biological replicates.

\section{Monitoring of Biofilm Formation}

Biofilm formation was carried out as described previously (Gutiérrez et al., 2016a). Briefly, standardized grown cultures were diluted down to $10^{7} \mathrm{CFU} / \mathrm{ml}$ in fresh TSBG broth (TSB supplemented with $0.25 \%$ glucose). Then, $100 \mu \mathrm{l}$ of this suspension were poured into 16 -well E-plates $\left(\sim 10^{6}\right.$ cells/well), which were then connected to the xCelligence RTCA-DP (ACEA Biosciences Inc., San Diego, CA, United States) holder prewarmed at $37^{\circ} \mathrm{C}$. Biofilm formation was monitored by recording impedance measurements of the CI every $10 \mathrm{~min}$ for $8 \mathrm{~h}$. Biofilm formation was performed in three independent biological replicates.

\section{Monitoring the Antibiofilm Effect of Phage-Derived Proteins}

The effect of phage-encoded proteins over 8 h-preformed biofilms was determined after addition of $100 \mu \mathrm{l}$ of increasing concentrations of the four phage-derived proteins $(0.04-12 \mu \mathrm{M})$ diluted in TSBG. Monitoring of the CI variations at $37^{\circ} \mathrm{C}$ was recorded every $10 \mathrm{~min}$ for an extra period of $16 \mathrm{~h}$. A sample with $\mathrm{NaPi}$ buffer $50 \mathrm{mM}$ pH $=7.4$ diluted in TSBG was also included as a control. The RTCA software 1.2.1 (ACEA Biosciences Inc.) was used for further analysis of the data obtained. First, a time-point normalization of the CI was performed $10 \mathrm{~min}$ after starting the treatment (at this time point, the value of the "normalized CI" is 1). These normalized CI values were used to calculate the percentage of biofilm removal referred to the control values after $16 \mathrm{~h}$ of treatment. In a next step, all data were referred to the control value ("baseline CI") by subtracting the normalized CI of each sample from the normalized CI of the control; thus, the value of the "baseline normalized CI" for the control is always 0 . From these data, the specific antibiofilm activity was determined as the decrease in the baseline normalized CI per $\mathrm{mM}$ of protein and per minute ( $\Delta$ baseline normalized $\mathrm{CI} \times \mathrm{mM}^{-1} \times \mathrm{min}^{-1}$ ) in the linear range of the curve. Moreover, these baseline normalized CI values were also used to calculate a dose-response curve (DRC) of the baseline normalized CI at a particular time $v s$ concentration (\% of baseline normalized CI $\times$ time $v s \mu \mathrm{M}$ of protein). The time was set according to the following two criteria: first, there should be a clear difference in the effect of the concentrations under study, and, second, the DRC should fit $R^{2}>0.98$ at the selected time point. The resultant sigmoidal DRC was calculated at this time for the three biological replicates independently; the $\mathrm{MBEC}_{50}$ was finally determined by the RTCA software and expressed as the mean \pm standard deviation. The $\mathrm{MBEC}_{50}$ was defined previously as the concentration causing 50\% reduction in the biofilm metabolic activity (Budzynska et al., 2011); herein, $\mathrm{MBEC}_{50}$ represents the minimum biofilm eradicating concentration of protein that decreases the normalized CI $\times$ time by $50 \%$. Moreover, the LOABE value was defined as the lowest concentration of protein tested that produced a detectable antibiofilm effect. This value is equivalent to the LOAEL (lowest observed adverse effect level) calculated in epidemiological or toxicological animal studies (Jeffery et al., 2004; Valdés et al., 2015).

Additionally, traditional staining with crystal violet was carried out to determine the total biomass adhered to the gold microelectrodes of the E-plate wells after protein treatment $(16 \mathrm{~h})$ with the modifications described previously (Gutiérrez et al., 2016a). The percentage of biofilm removal was calculated in reference to the control wells.

\section{Statistical Analysis}

The SPSS Statistics for Windows V. 22.0 (IBM Corp.) package was used to perform two assessments: (1) within each strain, antibiofilm differences among phage-derived proteins, and (2) within each phage-derived protein, antibiofilm differences among strains. The data were expressed as the mean \pm standard deviation and the differences were determined by one-way analysis of variance (ANOVA) followed by the StudentNewman-Keuls test for comparison of means at a level of significance $p<0.05$.

In addition, the LOABE value was calculated for each protein using the values obtained from DRC at the selected time point by comparing (two by two, by one-way ANOVA) the baseline normalized CI values obtained for consecutive protein concentrations; the LOABE was defined as the first point that showed statistical differences $(p<0.05)$.

On the other hand, linear regression equations among different numeric parameters (percentage of biofilm reduction calculated using normalized CI vs absorbance $495 \mathrm{~nm}$, and absorbance $495 \mathrm{~nm}$ vs specific antibiofilm activity) were calculated in order to obtain the coefficients of determination $\left(R^{2}\right)$ which show how well data fit to the linear regression equations.

\section{RESULTS}

\section{Real-Time Monitoring of Biofilm Disruption by Phage-Derived Proteins}

Based on our previous experience, strain S. aureus 15981 was chosen for the screening of the effective concentration range of four phage-derived proteins as antibiofilm agents: the three lytic proteins LysH5, CHAP-SH3b and HydH5-SH3b and the polysaccharide depolymerase Dpo7. Indeed, this strain is a strong biofilm former and had previously shown a good correlation 
between RTCA data and other methods (crystal violet staining and viable cell counting; Gutiérrez et al., 2016a).

To establish the concentration range for the four proteins under study, we monitored S. aureus 15981 biofilm disruption with the xCelligence RTCA system. To do that, biofilms were grown in the E-plates for $8 \mathrm{~h}$ until early stationary phase was reached. At this point, increasing concentrations (from 0.04 to $12 \mu \mathrm{M})$ of the four purified proteins were added to the wells. Immediately after adding the proteins, there is a noticeable increase in the impedance signal which seems to be proportional to the protein concentration. However, this increase was actually due to the buffer used for protein storage $(\mathrm{NaPi}, 50 \mathrm{mM}$ $\mathrm{pH}=7.4$ ), and not to protein activity. Indeed, treatment of $8 \mathrm{~h}$ old preformed biofilms with different dilutions of the buffer alone in TSB led to an initial increase in the impedance signal, followed by a stabilization of the values after $10 \mathrm{~min}$ (data not shown). As a result, to avoid the effect of the buffer and ensure that all the values obtained are protein dependent, normalization of the impedance signal was performed after $10 \mathrm{~min}$. Thus, at this time (10 min) data were transformed to "Baseline normalized CI" and measurements after this point, were used to calculate the activity of the different proteins (Supplementary Figure S1).

The treatment of 8-h biofilms of S. aureus 15981 with increasing concentrations of the phage-derived proteins resulted in a dose-dependent reduction of the baseline normalized CI due to biofilm removal (Figure 1A). The minimum value was reached after $2 \mathrm{~h}$ for LysH5 and CHAP-SH3b and 7-8 $\mathrm{h}$ for HysdH5/SH3b and Dpo7; these lowest baseline normalized CI values, indicating the lowest remnant biofilm biomass, correspond with the highest protein concentration. After this time point, all impedance values remained unaltered.

\section{Calculation of the $\mathrm{MBEC}_{50}$}

To compare the efficacy of the four proteins, a numerical parameter from the baseline normalized CI vs time curves was established after defining the best time point for each protein (dashed line, Figure 1A). Then, at this time, the baseline normalized $\mathrm{CI}$ values were represented as a function of the protein concentration resulting in a DRC (Figure 1B). The best time point to obtain the DRC was set according to two criteria: first, the effect shown by the protein concentrations should be clearly differentiated, and, second, the resulting DRC should fit $R^{2}>0.98$. The obtained results confirmed that an increase in protein concentration led to a gradual reduction in the normalized CI values and that this decrease was higher at higher concentrations of the compounds tested. Therefore, the DRCs clearly demonstrate a dose-dependent antibiofilm effect of the proteins tested against $8 \mathrm{~h}$-biofilms formed by $S$. aureus 15981. These curves subsequently allowed the calculation of the $\mathrm{MBEC}_{50}$ and the LOABE values corresponding to the four proteins (Figure 1B and Table 1). MBEC $_{50}$ values ranged from $3.5 \pm 1.1$ to $6.6 \pm 0.5 \mu \mathrm{M}$, obtained respectively for LysH5 and $\mathrm{HydH} 5-\mathrm{SH} 3 \mathrm{~b}$, and showed statistically significant differences among the four proteins tested $(p<0.05)$. Interestingly, the biofilm removal activity of the exopolysaccharide depolymerase Dpo7 and CHAP-SH3b was similar to that observed for LysH5 $(p \geq 0.05)$. Moreover, within the studied concentrations range, the LOABE values were similar for all four proteins $(\sim 1.5 \mu \mathrm{M})$ (Figure 1B and Table 1). This result seemed to indicate that concentrations below the LOABE values did not alter the biofilm structure, neither lysing the cells in the case of the lytic proteins, nor removing the polysaccharide matrix in the case of Dpo7.

To study a potential relationship between antimicrobial and antibiofilm activities for the phage lytic proteins (LysH5, CHAPSH3b, and HydH5-SH3b), we determined the specific lytic activity of each protein against $S$. aureus 15981 . The results of this assay revealed that LysH5 and CHAP-SH3b showed the highest specific lytic activity (2.5 and 1.7 units, respectively), while HydH5-SH3b exhibited a lower specific activity (0.6 units) (Table 1). This result is in good agreement with the lower antibiofilm activity observed by RTCA for this protein in comparison with the other two. Moreover, MIC values were further calculated and were surprisingly similar to the MBEC $_{50}$ values obtained for each protein (Table $\mathbf{1}$ ).

\section{Spectrum of Activity of Antibiofilm Proteins}

Our results, so far, confirmed the potential of the xCelligence RTCA system for screening antibiofilm compounds. In a step forward, this procedure was used to assess the spectrum of activity of the selected antibiofilm agents for other S. aureus isolates. Three additional staphylococcal strains with different ability to form biofilms were used: ISP479r is a strong biofilm producer and IPLA1 and Sa9 are weak biofilm producers. The ability of these strains to produce stronger (15981 and ISP479r) or weaker (Sa9 and IPLA1) biofilms was established after measuring the $\mathrm{CI}$ for $8 \mathrm{~h}$. In general, the maximum CI values for strong biofilm producers in stationary phase were over 2 whereas the values obtained for weak biofilm producers were around 1 (data not shown). Preformed biofilms (8 h-old) of $S$. aureus 15981, ISP479r, IPLA1, and Sa9 were treated with each protein for $16 \mathrm{~h}$ at $37^{\circ} \mathrm{C}$ using a protein concentration $(\sim 7 \mu \mathrm{M})$ which was twice the $\mathrm{MBEC}_{50}$ for LysH5, CHAP$\mathrm{SH} 3 \mathrm{~b}$ and Dpo7; in the case of HydH5-SH3b, the MBEC 50 value $(6.6 \mu \mathrm{M})$ was used due to the impossibility to purify a more concentrated stock of this last protein. The four assayed staphylococcal strains turned out to be sensitive to all proteins since a decrease in the baseline normalized CI can be observed in all cases (Figure 2). The baseline normalized CI data were then used to define the antibiofilm specific activity ( $\Delta$ baseline normalized $\mathrm{CI} \times \mathrm{mM}^{-1} \times \mathrm{min}^{-1}$ ), which resulted in values ranging from $0.08 \pm 0.01$ to $0.79 \pm 0.02$ units (Table 2). These values reflect the sensitivity of a given strain to each protein along time, allowing comparison of the activity spectrum among different proteins. Moreover, taking together the data obtained with different strains, we could establish similarities and differences in the activity spectra of the four proteins. When the data obtained for the four strains were combined, differences among the treatment with the four proteins $(p<0.05)$ were easily denoted. For instance, LysH5 showed the highest specific antibiofilm activity against all strains, while the lytic proteins derived from the VAPGH HydH5 possessed a medium specific antibiofilm activity. In contrast, the lowest specific antibiofilm 


\section{A}
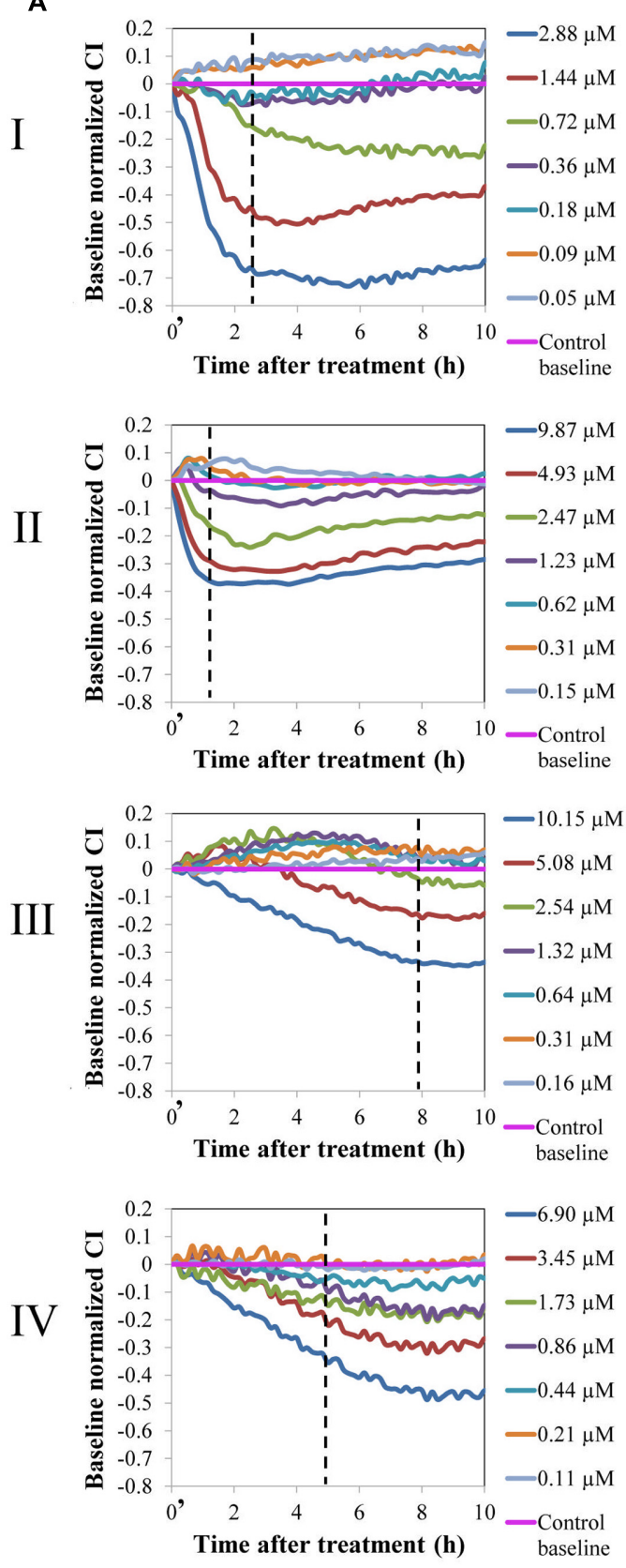

B
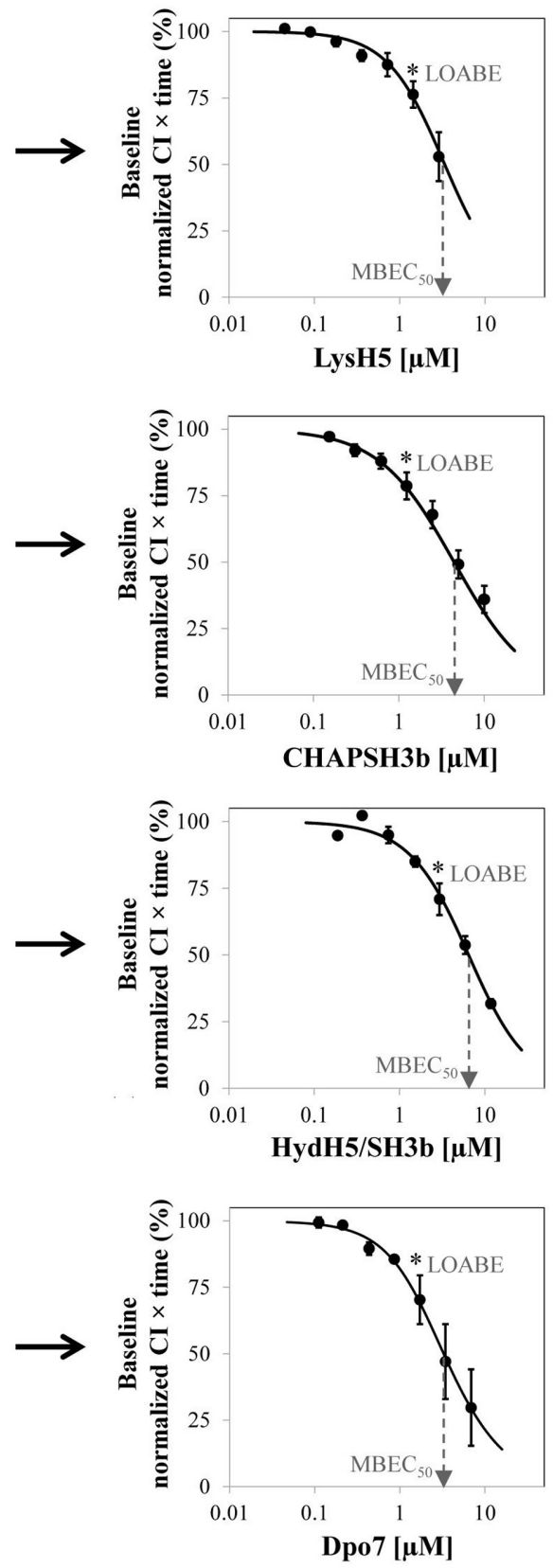

FIGURE 1 | Removal of $8 \mathrm{~h}$-old preformed biofilm of S. aureus 15981 treated with increasing concentrations of phage-derived proteins: LysH5 (I), CHAP-SH3b (II), $\mathrm{HydH5/SH3b}$ (III), and Dpo7 (IV). (A) Variation of the baseline normalized cell index (Cl) during biofilm treatment for one representative biological replicate out of three showing the same trend. Time $t=0^{\prime}$ in the $x$-axis represents the time 10 min after the beginning of the treatment. (B) Dose-response curve (DRC) fitted into a sigmoidal curve $\left(R^{2}>0.98\right)$ obtained by representing the baseline normalized $\mathrm{Cl}$ vs protein concentration at a particular time (dashed line in $\left.\mathbf{A}\right)$; each point in the curve corresponds with the mean ( \pm standard deviation) calculated from three biological replicates. The dashed arrow indicates the MBEC $\mathrm{C}_{50}$ value and the asterisk the LOABE value (see Table 1).

activity against all strains was observed for the exopolysaccharide depolymerase Dpo7 (Supplementary Figure S2). Interestingly, the activity of the proteins against the biofilms was not dependent on the robustness of the biofilm since similar values of activity were obtained against strong and weak biofilms for each protein (Table 2).
Finally, to confirm a correlation between the data obtained with the RTCA system and the standard crystal violet staining procedure, the percentage of biofilm reduction was calculated at the point corresponding to $16 \mathrm{~h}$ of treatment. On the one hand, the calculation was performed using the normalized CI values before adjusting the parameters to the control baseline 
TABLE 1 | Activity of phage-derived proteins.

\begin{tabular}{|c|c|c|c|c|}
\hline & \multicolumn{2}{|c|}{ Antibiofilm effect } & \multicolumn{2}{|l|}{ Lytic activity } \\
\hline & $\begin{array}{l}\text { MBEC }_{50} \\
(\mu \mathrm{M})\end{array}$ & $\begin{array}{l}\text { LOABE } \\
(\mu \mathrm{M})\end{array}$ & $\begin{array}{l}\text { Specific activity }(\Delta O D \\
\left.\quad \times \mu M^{-1} \times \min ^{-1}\right)\end{array}$ & $\begin{array}{l}\text { MIC } \\
(\mu \mathrm{M})\end{array}$ \\
\hline LysH5 & $3.5 \pm 1.1^{\mathrm{a}}$ & 1.4 & $2.5 \pm 0.5^{a}$ & 2.6 \\
\hline CHAP-SH3b & $4.4 \pm 1.2^{\mathrm{a}}$ & 1.2 & $1.7 \pm 0.6^{a}$ & 3.1 \\
\hline $\mathrm{HydH5/SH3b}$ & $6.6 \pm 0.5^{b}$ & 1.5 & $0.6 \pm 0.2^{b}$ & 5.4 \\
\hline Dpo7 & $3.7 \pm 1.5^{\mathrm{a}}$ & 1.7 & - & - \\
\hline
\end{tabular}

$M_{B E C_{50}}(\mu M)$ and LOABE $(\mu M)$ of the different proteins against $8 \mathrm{~h}$-old preformed biofilms of S. aureus 15981. The lytic activity of the proteins was calculated against planktonic cells and is expressed as specific lytic activity $\left(\Delta O D \times \mu M^{-1} \times\right.$ min $\left.^{-1}\right)$ and MIC $(\mu M)$. MBEC 50 and specific lytic activity values represent mean \pm standard deviation of three biological replicates. MIC is expressed as the mode of three independent biological repeats. Values in the same column having distinct letter are statistically different $(p<0.05)$ according to the ANOVA and SLK post hoc comparison test.

(Figure 3A) and, on the other hand, the total biomass was determined by crystal violet staining measuring the absorbance at $595 \mathrm{~nm}$ (Figure 3B). When comparing both results, in general, there were no statistically significant differences between them ( $p \geq 0.05)$ indicating that the RTCA system can be used to calculate the percentage of biofilm reduction, similarly to the standard crystal violet method currently used. Indeed, both methods are measuring the amount of remnant biomass after treatment with phage-related proteins at a given point. In fact, there was a good correlation between the data obtained with the two systems (Supplementary Figure S3). However, as expected, there was not a good correlation between the percentages of biofilm reduction calculated with crystal violet staining and the specific antibiofilm activity (data not shown). This is not surprising since the latter is representative of death kinetics where three parameters are taken into account: the decrease in impedance, the concentration of protein used in the experiment and the time spent to reach the lowest impedance value.

\section{DISCUSSION}

Over the last few years, the study of phage lytic proteins for the treatment of infectious diseases has attracted great interest (Fischetti, 2010). More recently, they have even been proposed as disinfectants for the food industry (Gutiérrez et al., 2016b). Indeed, there is evidence that many of these proteins are effective in animal models of infection due to the fact that they also have antimicrobial activity against biofilms formed by these bacteria
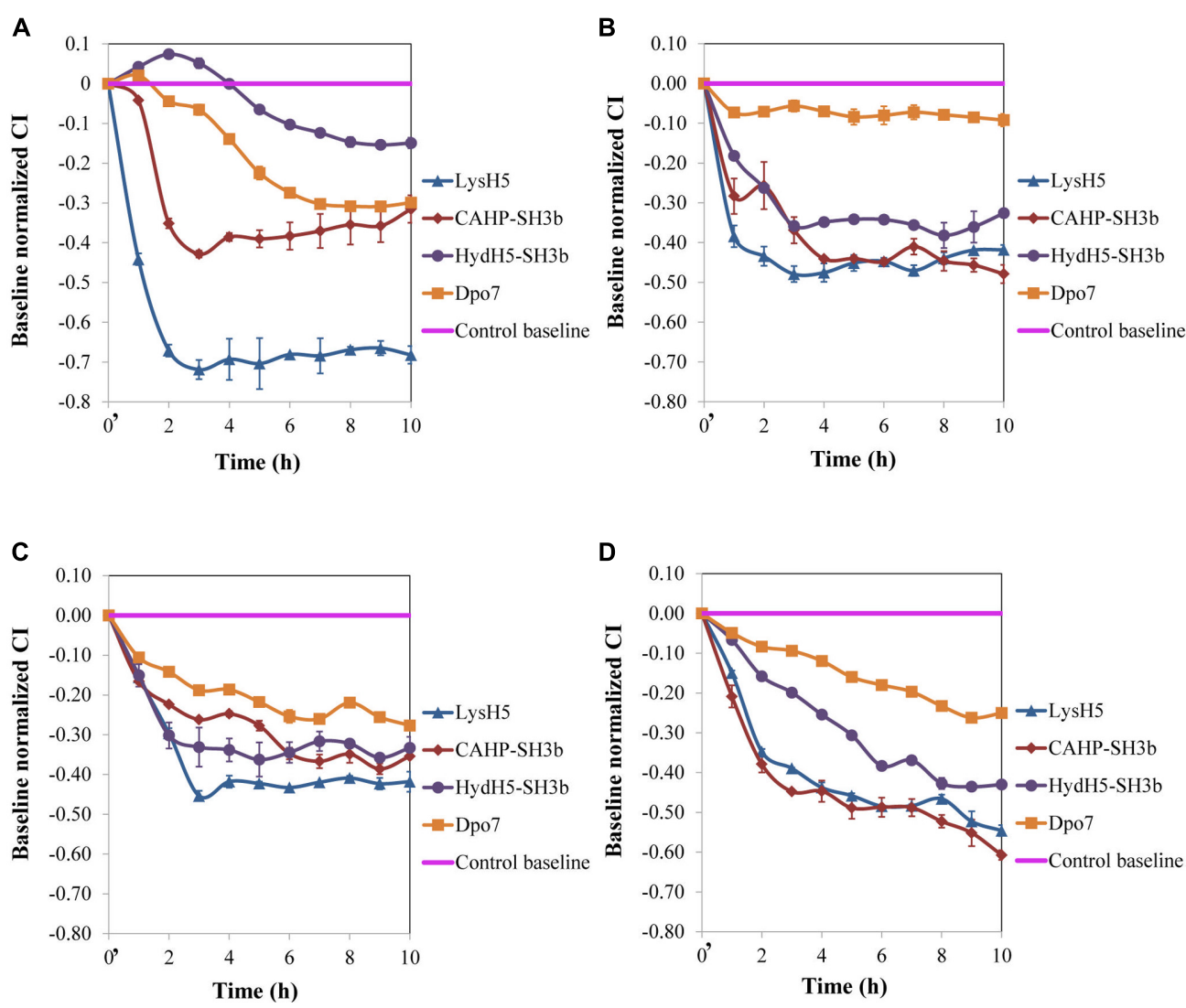

FIGURE 2 | Removal of 8 h-old preformed biofilms of S. aureus (A) 15981, (B) ISP479r, (C) IPLA1, and (D) Sa9, treated with $7 \mu$ M of proteins LysH5, CHAP-SH3b, HydH5-SH3b, and Dpo7. Mean \pm standard deviation was calculated for each value of normalized Cl throughout incubation from three biological replicates. Time $t=0^{\prime}$ in the $x$-axis represents the time 10 min after the beginning of the treatment. 
TABLE 2 | Specific antibiofilm activity ( $\Delta$ baseline normalized $\mathrm{Cl} \times \mathrm{mM}^{-1} \times \mathrm{min}^{-1}$ ) of the different proteins against $8 \mathrm{~h}-$ old biofilms formed by four S. aureus strains (15981, ISP479r, IPLA1, and Sa9).

\begin{tabular}{|c|c|c|c|c|}
\hline \multirow[t]{2}{*}{ Protein $(\mu \mathrm{M})$} & \multicolumn{4}{|c|}{ S. aureus strains } \\
\hline & 15981 & ISP479r & IPLA1 & Sa9 \\
\hline LysH5 (7 $\mu \mathrm{M})$ & $0.79 \pm 0.03^{a, A}$ & $0.47 \pm 0.04^{b, A}$ & $0.35 \pm 0.02^{c, A}$ & $0.41 \pm 0.02^{b, A}$ \\
\hline CHAP-SH3b $(7 \mu \mathrm{M})$ & $0.38 \pm 0.03^{a, B}$ & $0.21 \pm 0.03^{b, B}$ & $0.25 \pm 0.02^{\mathrm{c}, \mathrm{B}}$ & $0.45 \pm 0.01^{d, B}$ \\
\hline HydH5/SH3b $(7 \mu \mathrm{M})$ & $0.09 \pm 0.04^{a, c}$ & $0.28 \pm 0.01^{b, B}$ & $0.36 \pm 0.01^{\mathrm{c}, \mathrm{A}}$ & $0.18 \pm 0.01^{d, C}$ \\
\hline Dpo7 (7 $\mu \mathrm{M})$ & $0.11 \pm 0.01^{a, C}$ & $0.08 \pm 0.01^{b, c}$ & $0.15 \pm 0.01^{c, C}$ & $0.10 \pm 0.01^{d, D}$ \\
\hline
\end{tabular}

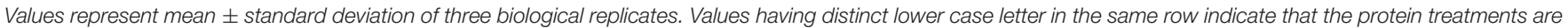

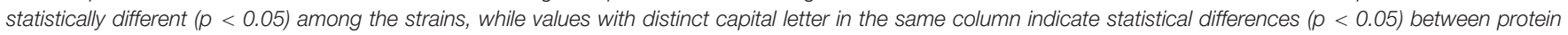
treatments within each strain, according to the ANOVA and SLK post hoc comparison test.
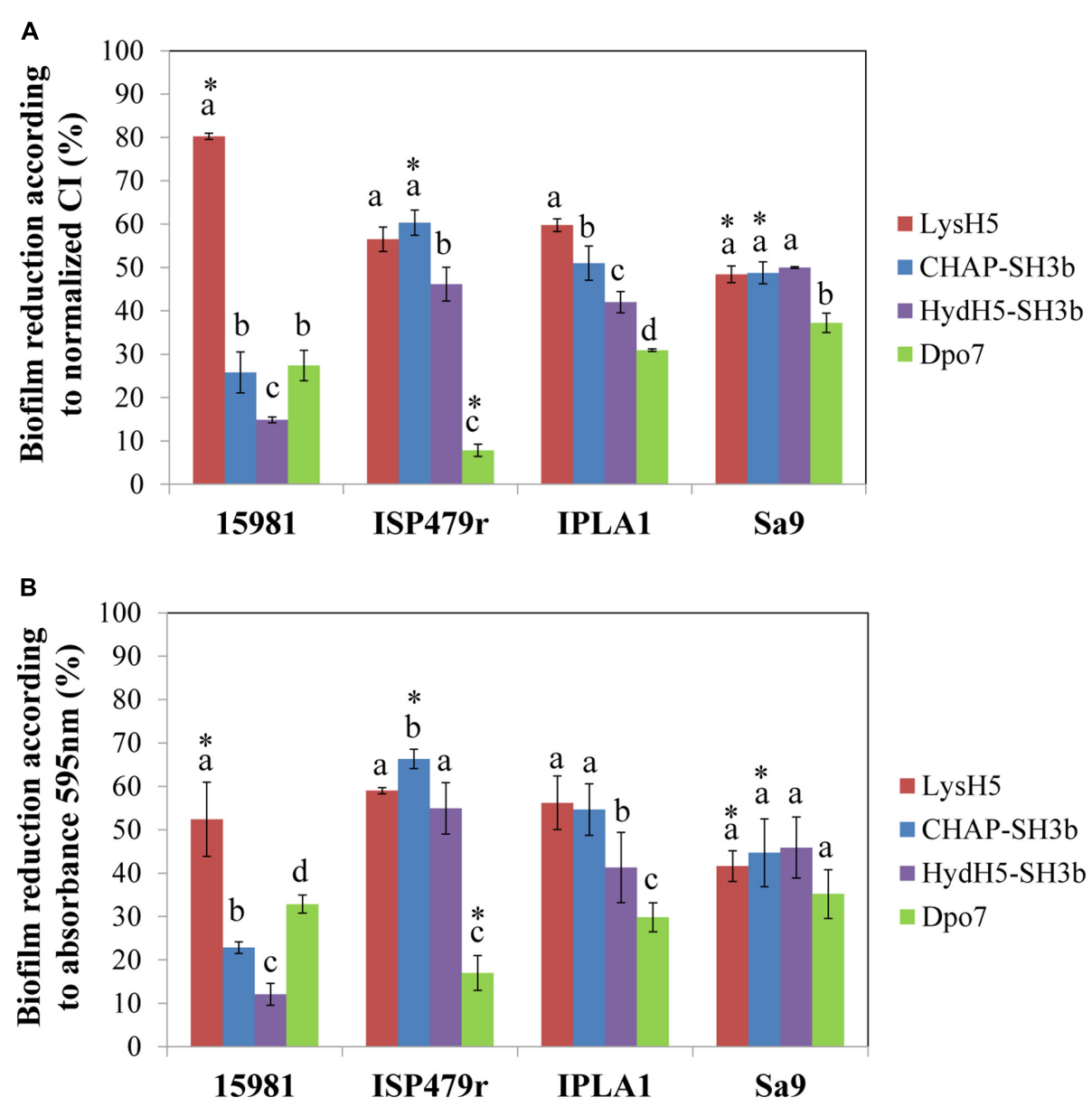

FIGURE 3 | Comparison of the RTCA-based technique and conventional crystal violet staining expressed as biofilm reduction (\%) after $16 \mathrm{~h}$ of treatment at $37^{\circ} \mathrm{C}$. Data were calculated for each biofilm-producing staphylococcal strain treated with the protein under study using the values obtained by measuring (A) the normalized Cl or (B) crystal violet staining (absorbance at $595 \mathrm{~nm}$ ). Bars represent mean \pm standard deviation of three biological replicates. Bars within each strain having distinct lower case letter indicate that the protein treatment is statistically different $(p<0.05)$; for the each pair strain/protein, an asterisk represents statistical differences between the biofilm reduction (\%) obtained using the normalized $\mathrm{Cl}$ and the absorbance $595 \mathrm{~nm}$ values $(p<0.05$; ANOVA and SLK post hoc comparison).

(Singh et al., 2010; Díez-Martínez et al., 2013; Schmelcher et al., 2015).

A major shortcoming in the study of biofilms is the lack of consensus among the diversity of techniques used to grow and study these structures. When working in static conditions, biofilm formation in microtiter plates is certainly the most common method used, to test both biofilm formation and the antibiofilm activity of different compounds. However, this technique involves endpoint quantification, and although amount of biofilm biomass, cell viability or metabolic activity 
can be determined by easy and cheap staining techniques, the results obtained sometimes are not reproducible and are person or laboratory dependent (Azeredo et al., 2017). Realtime monitoring would, however, overcome this issue as it allows quantification of changes throughout biofilm after treatment, being highly reproducible between laboratories (Gutiérrez et al., 2016a; Ferrer et al., 2017). The goal of this study was to design a method to determine the antibiofilm activity of phage-derived proteins against staphylococcal biofilms. This measurement should be useful for the assessment of both phage lytic proteins and other phage-derived proteins such as exopolysaccharide depolymerases. In addition, a definition of activity units was proposed to easily compare among different proteins. We had previously characterized several phage lytic proteins (CHAP$\mathrm{SH} 3 \mathrm{~b}$ and HydH5-SH3b) with enhanced staphylolytic activity, derived from the VAPGH HydH5 and lysostaphin (RodríguezRubio et al., 2012b). In addition, we determined that endolysin LysH5, encoded by the S. aureus phage vB_SauS-phiIPLA88, reduced staphylococcal sessile counts by 1-3 log units in polystyrene adhered biofilms (Gutiérrez et al., 2014). Similarly, the exopolysaccharide depolymerase Dpo7, encoded by the Staphylococcus epidermidis bacteriophage vB_SepiS-phiIPLA7 was able to remove up to $90 \%$ of biomass in staphylococcal biofilms formed by polysaccharide-producing strains (Gutiérrez et al., 2015). To date, the peptidoglycan hydrolase activity of phage lytic proteins is determined spectrophotometrically (turbidity reduction assay) and the definition of activity units is based on the decrease of optical density. Thus, one enzymatic unit would represent the amount of protein able to reduce the $\mathrm{OD}_{600}$ of a bacterial suspension by $50 \%$ in 15 min (Briers et al., 2007). Moreover, the reduction in optical density over time (minutes or hours) can be used to calculate the hydrolysis rate known as "specific lytic activity" that is reported as $\Delta \mathrm{OD} \times \mathrm{time}^{-1} \times \mathrm{mg}^{-1}$ of lytic protein. In addition to the turbidity reduction method, there are other assays such as time-kill curve (viable counts), zymogram, spot-on-lawn, MIC and minimum bactericidal concentration, that can be performed to test the lytic activity of phage proteins (Nelson et al., 2012). For measuring exopolysaccharide depolymerase activity against bacterial biofilms there is no standardized approach beyond the reduction in total biomass of biofilms quantified by crystal violet staining (Gutiérrez et al., 2015). A different approach is the measurement of $\beta$-hexosaminidase activity of the bacterial exopolysaccharide depolymerase DspB against a synthetic substrate (Kaplan et al., 2003).

Here, we used the RTCA methodology to infer the decrease in impedance as a direct measurement of antibiofilm activity. Previously, the impedance-based system proved to be an accurate technology to measure the ability of staphylococcal strains to form biofilm, showing a high correlation with values obtained by standard approaches such as crystal violet staining and bacterial cell counts, as well as with those obtained upon other abiotic surfaces (polystyrene and stainless steel; Gutiérrez et al., 2016a). The RTCA method was also validated for biofilm removal using bacteriophages and phage-derived proteins (Gutiérrez et al., 2016a) and some antibiotics (Ferrer et al., 2017).
In the current work, we have shown that this methodology is useful to perform a reliable and quick screening of proteins with antibiofilm activity and also to test the sensitivity of different staphylococcal strains to these proteins. Our results showed $\mathrm{MBEC}_{50}$ values in the micromolar scale $(3.5 \mu \mathrm{M})$ for LysH5, CHAP-SH3b, and Dpo7. As expected, the highest $\mathrm{MBEC}_{50}$ values against $S$. aureus 15981 were obtained for HydH5-SH3b that showed the lowest antimicrobial activity against planktonic cells. This chimeric protein also had lower lytic activity against planktonic cells of another S. aureus Sa9 strain (Rodríguez-Rubio et al., 2012a). However, this correlation does not necessarily imply that antibiofilm activity is equivalent to lytic activity. For instance, diffusion of the protein into the biofilm, which is determined by size and charge, would limit its activity (Zhang et al., 2011). This limited activity due to diffusion of the protein, is also highlighted due to the $\mathrm{MBEC}_{50}$ values obtained for the lytic proteins were equal to onefold the MIC, indicating that a higher concentration of the protein is needed to remove biofilms. Similar results were obtained using a novel antimicrobial compound (mul-1867) against Pseudomonas aeruginosa biofilms, where a concentration of onefold the MIC was able to remove the $50 \%$ of the biofilm (Tetz et al., 2016).

\section{CONCLUSION}

In conclusion, the method proposed here will allow the easy screening, in static conditions, of specific settings for biofilm removal ( $\mathrm{pH}$, temperature, ionic strength). Moreover, a high number of strains can be simultaneously tested for their sensitivity to the proteins since there are several RTCA systems available on the market that allow testing up to 1536 samples in the same experiment. Taking into account the small size of the equipment, the RTCA device can easily fit into any incubator to perform experiments at different temperatures (ranging from 15 to $40^{\circ} \mathrm{C}$ ). It is worth noting that, to make the most of this technology, it would be helpful to develop the RTCA $\mathrm{xCelligence} \mathrm{systems} \mathrm{to} \mathrm{allow} \mathrm{for} \mathrm{testing} \mathrm{of} \mathrm{biofilm} \mathrm{formation}$ and removal under dynamic conditions. Unfortunately, to date this technology only allows testing under static conditions. Of note, the real-time recording of impedance values allows determining the precise time where the maximum elimination of the biofilm is achieved, and it is also suitable for the calculation of parameters as important as the $\mathrm{MBEC}_{50}$ and $\mathrm{LOABE}$ values, which represent the protein concentration needed to eliminate the $50 \%$ of the biofilm and the minimum concentration to produce an alteration in the biofilm, respectively. This technique also permits determining the specific antibiofilm activity, which is an indicator of biofilm degradation dependent on the incubation time and on the protein concentration. The results obtained in this study show that the percentage of biofilm removal values estimated with RTCA correlate with those obtained using a standard staining method, while providing additional information. Taken together, the values of the effective dose for biofilm removal and the spectrum of activity against a strain collection facilitate the evaluation of antibiofilm properties of these proteins. This would also be an advantage 
to speed up the process by selecting optimal combinations of phage lytic proteins, exopolysaccharide depolymerases or other antimicrobial compounds against biofilms. In this sense, the study of such synergistic effects should be a focus of further research.

\section{AUTHOR CONTRIBUTIONS}

DG, LF, BM, PR-M, PG, and AR conceived and designed the experiments. DG performed the experiments. DG, PR-M, PG, and AR analyzed the data. DG, LF, BM, PR-M, PG, and AR wrote the paper.

\section{FUNDING}

This study was supported by grants AGL2015-65673-R (Program of Science, Technology and Innovation 2013-2017, Ministry of Science and Innovation, Spain), GRUPIN14-139 (Program of

\section{REFERENCES}

Anand, S., and Singh, D. (2013). Resistance of the constitutive microflora of biofilms formed on whey reverse-osmosis membranes to individual cleaning steps of a typical clean-in-place protocol. J. Dairy Sci. 96, 6213-6222. doi: $10.3168 /$ jds.2013-7012

Azeredo, J., Azevedo, N. F., Briandet, R., Cerca, N., Coenye, T., Costa, A. R., et al. (2017). Critical review on biofilm methods. Crit. Rev. Microbiol. 43, 313-351. doi: 10.1080/1040841X.2016.1208146

Bjarnsholt, T. (2013). The role of bacterial biofilms in chronic infections. APMIS Suppl. 121, 1-58. doi: 10.1111/apm.12099

Bridier, A., Sánchez-Vizuete, P., Guilbaud, M., Piard, J. C., Naitali, M., and Briandet, R. (2015). Biofilm-associated persistence of food-borne pathogens. Food Microbiol. 45, 167-178. doi: 10.1016/j.fm.2014.04.015

Briers, Y., Volckaert, G., Cornelissen, A., Lagaert, S., Michiels, C. W., Hertveldt, K., et al. (2007). Muralytic activity and modular structure of the endolysins of Pseudomonas aeruginosa bacteriophages phiKZ and EL. Mol. Microbiol. 65, 1334-1344. doi: 10.1111/j.1365-2958.2007.05870.x

Budzynska, A., Wieckowska-Szakiel, M., Sadowska, B., Kalemba, D., and Rozalska, B. (2011). Antibiofilm activity of selected plant essential oils and their major components. Pol. J. Microbiol. 60, 35-41.

CLSI (2015). Performance Standards for Antimicrobial Susceptibility Testing; Twenty-Fifth Informational Supplement. CLSI Approved Document M100-S25. Wayne, PA: Clinical and Laboratory Standards Institute.

Díez-Martínez, R., de Paz, H. D., Bustamante, N., García, E., Menéndez, M., and García, P. (2013). Improving the lethal effect of cpl-7, a pneumococcal phage lysozyme with broad bactericidal activity, by inverting the net charge of its cell wall-binding module. Antimicrob. Agents Chemother. 57, 5355-5365. doi: 10.1128/AAC.01372-13

ECDC (2013). Point Prevalence Survey of Healthcare-Associated Infections and Antimicrobial Use in European Acute Care Hospitals. Stockholm: ECDC.

EFSA and ECDC (2016). The European Union summary report on trends and sources of zoonoses, zoonotic agents and food-borne outbreaks in 2014. EFSA J. 13:4329. doi: 10.2903/j.efsa.2015.4329

Ferrer, M. D., Rodríguez, J. C., Álvarez, L., Artacho, A., Royo, G., and Mira, A. (2017). Effect of antibiotics on biofilm inhibition and induction measured by real-time cell analysis. J. Appl. Microbiol. 122, 640-650. doi: 10.1111/jam.13368

Fischetti, V. A. (2010). Bacteriophage endolysins: a novel anti-infective to control Gram-positive pathogens. Int. J. Med. Microbiol. 300, 357-362. doi: 10.1016/j. ijmm.2010.04.002

Flemming, H. C., and Wingender, J. (2010). The biofilm matrix. Nat. Rev. Microbiol. 8, 623-633. doi: 10.1038/nrmicro2415
Science, Technology and Innovation, Principado de Asturias, Spain and EU FEDER funds), and BLAAT ID 67 (ANIWHA ERA-Net EC Programme). LF was awarded a "Marie Curie Clarin-Cofund" grant. PG, BM, and AR are members of the FWO Vlaanderen funded "Phagebiotics" research community (WO.016.14) and the bacteriophage network FAGOMA.

\section{ACKNOWLEDGMENT}

We thank Dr. A. Toledo-Arana (Instituto de Agrobiotecnología, CSIC, Universidad Pública de Navarra, Spain) for providing the S. aureus 15981 and ISP479r strains.

\section{SUPPLEMENTARY MATERIAL}

The Supplementary Material for this article can be found online at: http://journal.frontiersin.org/article/10.3389/fmicb. 2017.01632/full\#supplementary-material

García, P., Madera, C., Martínez, B., and Rodríguez, A. (2007). Biocontrol of Staphylococcus aureus in curd manufacturing processes using bacteriophages. Int. Dairy J. 17, 1232-1239. doi: 10.1016/j.idairyj.2007.03.014

García, P., Martínez, B., Rodríguez, L., and Rodríguez, A. (2010). Synergy between the phage endolysin LysH5 and nisin to kill Staphylococcus aureus in pasteurized milk. Int. J. Food Microbiol. 141, 151-155. doi: 10.1016/j. ijfoodmicro.2010.04.029

Gutiérrez, D., Briers, Y., Rodríguez-Rubio, L., Martínez, B., Rodríguez, A., Lavigne, R., et al. (2015). Role of the pre-neck appendage protein (Dpo7) from phage vB_SepiS-phiIPLA7 as an anti-biofilm agent in staphylococcal Species. Front. Microbiol. 6:1315. doi: 10.3389/fmicb.2015.01315

Gutiérrez, D., Delgado, S., Vázquez-Sánchez, D., Martínez, B., Cabo, M. L., Rodríguez, A., et al. (2012). Incidence of Staphylococcus aureus and analysis of associated bacterial communities on food industry surfaces. Appl. Environ. Microbiol. 78, 8547-8554. doi: 10.1128/AEM.02045-12

Gutiérrez, D., Hidalgo-Cantabrana, C., Rodríguez, A., García, P., and RuasMadiedo, P. (2016a). Monitoring in real time the formation and removal of biofilms from clinical related pathogens using an impedance-based technology. PLoS ONE 11:e0163966. doi: 10.1371/journal.pone.0163966

Gutiérrez, D., Rodríguez-Rubio, L., Martínez, B., Rodríguez, A., and García, P. (2016b). Bacteriophages as weapons against bacterial biofilms in the food industry. Front. Microbiol. 7:825. doi: 10.3389/fmicb.2016. 00825

Gutiérrez, D., Ruas-Madiedo, P., Martínez, B., Rodríguez, A., and García, P. (2014). Effective removal of staphylococcal biofilms by the endolysin LysH5. PLoS ONE 9:e107307. doi: 10.1371/journal.pone.0107307

Hall-Stoodley, L., and Stoodley, P. (2009). Evolving concepts in biofilm infections. Cell Microbiol. 11, 1034-1043. doi: 10.1111/j.1462-5822.2009.01323.x

Jeffery, B., Barlow, T., Moizer, K., Paul, S., and Boyle, C. (2004). Amnesic shellfish poison. Food Chem. Toxicol. 42, 545-557. doi: 10.1016/j.fct.2003.11.010

Kaplan, J. B., Ragunath, C., Ramasubbu, N., and Fine, D. H. (2003). Detachment of Actinobacillus actinomycetemcomitans biofilm cells by an endogenous betahexosaminidase activity. J. Bacteriol. 185, 4693-4698. doi: 10.1128/JB.185.16. 4693-4698.2003

Martin, M., Holscher, T., Dragos, A., Cooper, V. S., and Kovacs, A. T. (2016). Laboratory evolution of microbial interactions in bacterial biofilms. J. Bacteriol. 198, 2564-2571. doi: 10.1128/JB.01018-15

Miquel, S., Lagrafeuille, R., Souweine, B., and Forestier, C. (2016). Anti-biofilm activity as a health issue. Front. Microbiol. 7:592. doi: 10.3389/fmicb.2016.00592

Montanaro, L., Poggi, A., Visai, L., Ravaioli, S., Campoccia, D., Speziale, P., et al. (2011). Extracellular DNA in biofilms. Int. J. Artif. Organs 34, 824-831. doi: 10.5301/ijao.5000051 
Myszka, K., and Czaczyk, K. (2011). Bacterial biofilms on food contact surfaces - a review. Pol. J. Food Nutr. Sci. 61, 173-180. doi: 10.2478/v10222-011-0018-4

Nelson, D. C., Schmelcher, M., Rodriguez-Rubio, L., Klumpp, J., Pritchard, D. G., Dong, S., et al. (2012). Endolysins as antimicrobials. Adv. Virus Res. 83, 299-365. doi: 10.1016/B978-0-12-394438-2.00007-4

Obeso, J. M., Martínez, B., Rodríguez, A., and García, P. (2008). Lytic activity of the recombinant staphylococcal bacteriophage PhiH5 endolysin active against Staphylococcus aureus in milk. Int. J. Food Microbiol. 128, 212-218. doi: 10.1016/ j.ijfoodmicro.2008.08.010

O'Flaherty, S., Ross, R. P., and Coffey, A. (2009). Bacteriophage and their lysins for elimination of infectious bacteria. FEMS Microbiol. Rev. 33, 801-819. doi: 10.1111/j.1574-6976.2009.00176.x

Oliveira, H., Thiagarajan, V., Walmagh, M., Sillankorva, S., Lavigne, R., NevesPetersen, M. T., et al. (2014). A thermostable Salmonella phage endolysin, Lys68, with broad bactericidal properties against gram-negative pathogens in presence of weak acids. PLOS ONE 9:e108376. doi: 10.1371/journal.pone. 0108376

Olsen, I. (2015). Biofilm-specific antibiotic tolerance and resistance. Eur. J. Clin. Microbiol. Infect. Dis. 34, 877-886. doi: 10.1007/s10096-015-2323-z

Otto, M. (2013). Staphylococcal infections: mechanisms of biofilm maturation and detachment as critical determinants of pathogenicity. Annu. Rev. Med. 64, 175-188. doi: 10.1146/annurev-med-042711-140023

Pires, D. P., Oliveira, H., Melo, L. D., Sillankorva, S., and Azeredo, J. (2016). Bacteriophage-encoded depolymerases: their diversity and biotechnological applications. Appl. Microbiol. Biotechnol. 100, 2141-2151. doi: 10.1007/s00253015-7247-0

Rabin, N., Zheng, Y., Opoku-Temeng, C., Du, Y., Bonsu, E., and Sintim, H. O. (2015). Biofilm formation mechanisms and targets for developing antibiofilm agents. Future Med. Chem. 7, 493-512. doi: 10.4155/fmc.15.6

Rodríguez-Rubio, L., Gutiérrez, D., Donovan, D. M., Martínez, B., Rodríguez, A., and García, P. (2015). Phage lytic proteins: biotechnological applications beyond clinical antimicrobials. Crit. Rev. Biotechnol. 36, 542-552. doi: 10.3109/ 07388551.2014.993587

Rodríguez-Rubio, L., Gutiérrez, D., Martínez, B., Rodríguez, A., Gotz, F., and García, P. (2012a). The tape measure protein of the Staphylococcus aureus bacteriophage vB_SauS-phiIPLA35 has an active muramidase domain. Appl. Environ. Microbiol. 78, 6369-6371. doi: 10.1128/AEM. 01236-12

Rodríguez-Rubio, L., Martínez, B., Rodríguez, A., Donovan, D. M., and García, P. (2012b). Enhanced staphylolytic activity of the Staphylococcus aureus bacteriophage vB_SauS-phiIPLA 88 HydH5 virion-associated peptidoglycan hydrolase: fusions, deletions, and synergy with LysH5. Appl. Environ. Microbiol. 78, 2241-2248. doi: 10.1128/AEM.07621-11

Sass, P., and Bierbaum, G. (2007). Lytic activity of recombinant bacteriophage phil1 and phi12 endolysins on whole cells and biofilms of Staphylococcus aureus. Appl. Environ. Microbiol. 73, 347-352. doi: 10.1128/AEM.01616-06
Schmelcher, M., Powell, A. M., Camp, M. J., Pohl, C. S., and Donovan, D. M. (2015). Synergistic streptococcal phage lambdaSA2 and B30 endolysins kill streptococci in cow milk and in a mouse model of mastitis. Appl. Microbiol. Biotechnol. 99, 8475-8486. doi: 10.1007/s00253-015-6579-0

Shen, Y., Koller, T., Kreikemeyer, B., and Nelson, D. C. (2013). Rapid degradation of Streptococcus pyogenes biofilms by PlyC, a bacteriophageencoded endolysin. J Antimicrob. Chemother. 68, 1818-1824. doi: 10.1093/jac/ dkt104

Singh, R., Ray, P., Das, A., and Sharma, M. (2010). Penetration of antibiotics through Staphylococcus aureus and Staphylococcus epidermidis biofilms. J. Antimicrob, Chemother. 65, 1955-1958. doi: 10.1093/jac/dkq257

Speziale, P., Pietrocola, G., Foster, T. J., and Geoghegan, J. A. (2014). Proteinbased biofilm matrices in Staphylococci. Front. Cell. Infect. Microbiol. 4:171. doi: 10.3389/fcimb.2014.00171

Tetz, G., Vikina, D., and Tetz, V. (2016). Antimicrobial activity of mul-1867, a novel antimicrobial compound, against multidrug-resistant Pseudomonas aeruginosa. Ann. Clin. Microbiol. Antimicrob. 15, 19. doi: 10.1186/s12941-0160134-4

Valdés, L., Gueimonde, M., and Ruas-Madiedo, P. (2015). Monitoring in real time the cytotoxic effect of Clostridium difficile upon the intestinal epithelial cell line HT29. J. Microbiol. Methods 119, 66-73. doi: 10.1016/j.mimet.2015.09.022

Valle, J., Toledo-Arana, A., Berasain, C., Ghigo, J. M., Amorena, B., Penades, J. R., et al. (2003). SarA and not sigmaB is essential for biofilm development by Staphylococcus aureus. Mol. Microbiol. 48, 1075-1087. doi: 10.1046/j.1365-2958. 2003.03493.x

Van Acker, H., Van Dijck, P., and Coenye, T. (2014). Molecular mechanisms of antimicrobial tolerance and resistance in bacterial and fungal biofilms. Trends Microbiol. 22, 326-333. doi: 10.1016/j.tim.2014.02.001

Van Houdt, R., and Michiels, C. W. (2010). Biofilm formation and the food industry, a focus on the bacterial outer surface. J. Appl. Microbiol. 109, 1117-1131. doi: 10.1111/j.1365-2672.2010.04756.x

Zhang, Z., Nadezhina, E., and Wilkinson, K. J. (2011). Quantifying diffusion in a biofilm of Streptococcus mutans. Antimicrob. Agents Chemother. 55, 1075-1081. doi: 10.1128/AAC.01329-10

Conflict of Interest Statement: The authors declare that the research was conducted in the absence of any commercial or financial relationships that could be construed as a potential conflict of interest.

Copyright (C) 2017 Gutiérrez, Fernández, Martínez, Ruas-Madiedo, García and Rodriguez. This is an open-access article distributed under the terms of the Creative Commons Attribution License (CC BY). The use, distribution or reproduction in other forums is permitted, provided the original author(s) or licensor are credited and that the original publication in this journal is cited, in accordance with accepted academic practice. No use, distribution or reproduction is permitted which does not comply with these terms. 